\title{
RETOS Y PROSPECTIVAS HACIA UNA NUEVA POLÍTICA PÚBLICA FEMINISTA EN PUERTO RICO ${ }^{1}$
}

\author{
Zuleika Vidal Rodríguez ${ }^{2}$
}

\section{Introducción:}

En Puerto Rico se han desarrollado piezas legislativas en el área de seguridad Social y protección social a la mujer, en los últimos cincuenta años. Por otra parte, la población femenina ha ido en aumento, demográficamente, al igual que los problemas sociales que han impactado dramáticamente la calidad de vida de nuestras mujeres. Se ha reconocido que aún el aumento en medidas legislativas ó de seguridad social en general, no ha aumentado el poder societal de esta gran población del género femenino, todavía excluida en su mayoría. Asimismo, son notables los problemas sociales de: violencia doméstica, discrimen laboral, hostigamiento sexual y desprotección social en ausencia de política pública a las trabajadoras del hogar, mal llamadas "amas de casa". Por otro lado, la violencia doméstica asesina a un promedio de sesenta mujeres anualmente en Puerto Rico (Policía de Puerto Rico, 2002).

El problema central a ser abordado en este artículo gravita en la ausencia de una política feminista comprehensiva de las necesidades y problemas actuales de la mujer puertorriqueña moderna, siendo aún las mujeres un grupo excluido y carente de poder real en las estratas de servicios, y en la formulación de la política pública. Este problema cobra vigencia debido a la marginación en que viven en Puerto Rico por lo menos $1,250,000$ mujeres trabajadoras en el hogar, sin remuneración alguna o beneficios marginales o de cobertura en seguridad social (US Bureau of the Census, 2001). Su pertinencia para el trabajo social reconceptualizado, radica en la continua exclusión de las mujeres, de nuestros gobernantes y de los programas y servicios gubernamentales

\footnotetext{
${ }^{1}$ Artículo basado en la Ponencia presentada en el V Encuentro de Política Social y Trabajo Social celebrado en la Universidad de Costa Rica, 27-29 de mayo de 2003. ${ }^{2}$ Catedrática, Departamento de Trabajo Social, Universidad de Puerto Rico.
} 
que les compete a atender los graves problemas sociales y necesidades actuales de este sector. Las políticas sociales que se han instaurado, algunas relevantes, otras carentes de real impacto en esta población no han sido respaldadas en la mayoría de sus áreas, por servicios de seguridad social. El género femenino aunque mayoritario, ha disminuido en su calidad de vida, colocándolo en condición de esclavismo y marginalidad. Un sector del género femenino, la trabajadora con doble tarea, tiene escasez de servicios que inciden en la raíz de sus verdaderos problemas de desigualdad, por desempeñarse en un empleo y tener la responsabilidad de las tareas del hogar.

El trabajo social, debe estar inmerso en la defensa valiente de los derechos humanos y valores de salvaguardia a la dignidad del ser humano. Se requiere militancia en luchas de acción social en pro de la justicia social, en organización comunitaria para promover la calidad de vida de la familia y la sociedad. La mujer como sector poblacional ha estado invisibilizada de estas defensas, aún constituyendo nuestra principal cliente en términos cualitativos y cuantitativos. Es ella la que acude a solicitar servicios para la familia, los hijos/as en escuelas, hospitales, servicios a impedidos, familiares y de salud mental. No obstante, analizándonos críticamente como profesión en muchas instancias no hemos comprendido ni defendido asertivamente sus derechos y necesidades reales, como sector excluido.

El trabajo social reconceptualizado debe acercarse a las poblaciones excluidas, como la mujer; marginadas y con poco poder real, con nuevos enfoques comunitarios y de políticas públicas que ataquen los problemas y llenen las necesidades de la vida en el objetivo continuo de aumentar, a una calidad de convivencia integral y enriquecida. Los estudios del género, en investigación social y académica han estado relegados y muy poco abordados en sus consecuencias reales en la elaboración de políticas sociales vigorosas y de vanguardia para nuestra mayoritaria población de clientela, y de nuestra comunidad. Su aporte a nuevos enfoques filosóficos de calidad de vida y cuidado integral, con su repercusión en novedosas políticas públicas, impacto en servicios que faciliten la vida de las mujeres y sus familias ante el modernismo ha sido poco consistente. La teoría en trabajo social ha 
estado limitada, por su énfasis en la práctica. Teoría y práctica deben vincularse más cualitativamente con nuevos acercamientos metodológicos al ofrecerse los servicios no asistenciales de bienestar social, enfrentándonos ante la realidad puertorriqueña colonial de servicios decadentes ante el nuevo Estado Benefactor Neoliberal. Su aportación al crecimiento teórico - epistemológico se hace ya evidente y urgente si queremos prevalecer en el nuevo siglo como profesión que aboga por la justicia social. Las prácticas sociales tienen que revaluarse ante la demanda de justicia y los tiempos (Carpio \& Novacovsky, 1999).

Un sector de la población femenina en Puerto Rico ha avanzado en el pasado siglo en conquistas educativas y accesos a niveles universitarios. No obstante, el adelanto educativo no ha impactado significativamente a la fuerza laboral en procesos de poder. Las transformaciones de economía industrial débil y plagada de problemas inherentes al capitalismo, y coloniaje, han agravado más aún la situación de la mujer ante demandas crecientes sociales, y carencias de redes de apoyo institucionales y gubernamentales para adelantar su pesada carga de trabajo.

La mujer en Puerto Rico experimenta una sociedad tradicional con un sistema patriarcal que carga una socialización sexista en todas las instalaciones sociales (Vidal, 2001:25-30). La mujer puertorriqueña ha descendido económicamente, producto de la feminización de la pobreza en una jefatura de familia creciente estadísticamente, con connotaciones de sobrecarga al cuido y crianza de los hijos(as) y dificultades económicas por descenso en estrata social luego de separación o divorcio (Burgos y Colberg, 1992; Vidal, 2001). Esta situación se agrava ante la ausencia de política sobre educación sexual en el sector escolar en las jóvenes y un creciente aumento en embarazos de jóvenes.

Las políticas federales del neoliberalismo invisibilizan la situación real de la mujer menos educada, dependiente hasta el presente de programas de bienestar social con fondos federales, debido a situación de coloniaje político existente en Puerto Rico. Se le responsabiliza por su autogestión y suficiencia económica y aún, hay disposiciones expresas para que se case a fin de recibir ayudas en algunos programas 
como por ejemplo las amparadas en la ley de Reconciliación de Responsabilidad Personal y Oportunidad Laboral de 1996. Esta ley coexiste coetáneamente en un sistema republicano conservador, que agudiza aún más la difícil situación económica de las jefes de familia ante nuestra débil economía insular, plagada de desempleo y cierres continuos de industrias (Segarra, 2000).

Es necesario como reto, que el trabajo social asuma una postura proactiva y de apoderamiento de su propia clase profesional, para entonces atacar la injusticia social y crearse medidas estatales de seguridad social nativas en Puerto Rico. El estado neoliberal no lo hará; las clientes están muchas en desconocimiento de los recortes federales y de la nueva presión para la "autogestión" sin poseer medios materiales para su emancipación económica y personal. Tenemos el reto de ayudar a las mujeres a organizarse para lograr, dentro de las limitaciones de una política pública reaccionaria, la creación de otras alternativas, tales como cooperativas de mujeres jefas.

\section{Una ponderación de necesidades en etiología de problemas que enfrenta la mujer puertorriqueña}

La situación de la mujer en Puerto Rico se ha agravado a una calidad de vida deteriorada con ausencia de poder real en instituciones de las que forma parte y con políticas públicas que no responden a sus necesidades. Hay discrimen y estereotipación en algunas de estas políticas y falta de ofrecimiento de servicios en otras. Los problemas que experimenta la mujer en Puerto Rico (violencia familiar, discriminación laboral, doble jornal de trabajo, falta de toma control de su sexualidad y otros) son consecuencias y no causas del sistema sexista patriarcal. La situación puede resumirse en:

* Grupo mayoritario de mujeres desempleadas como "amas de casa" sin remuneración alguna o servicios de seguridad social 1,250,000 (Bureau of the Census, 2001). Fuerza laboral con sólo el 35.1 por ciento aumentando sólo el 4.1 por ciento, a pesar del aumento considerado de un sector femenino educado con grados universitarios. Los datos presentados a continuación apuntan a esta dirección. 
Un perfil de Mujer trabajadora asalariada a 2002 refleja:

Departamento del Trabajo de Puerto Rico,

Informe Estadístico (2001-2002)

Tasa de desempleo

Grupo de edades promedio fluctúa de

Escolaridad promedio

Nivel status social
11.4 por ciento

25-54 años

13 años o más

casada

Mayoría empleada pública, tres primeros tipos prioritarios estadísticamente en: trabajadoras de oficina; trabajadoras profesionales relacionadas como maestras, semiprofesionales, técnicas de salud; administradoras, oficiales y relacionado

Violencia

Doméstica

Divorcios
20,000 casos radicados ante el Tribunal, ley

54,60 casos promedio asesinatos de mujeres

dos de cada tres matrimonios se divorcian. Con la segunda tasa más alta en el mundo

$\underline{\text { Investigaciones sociales revelantes al discrimen por género }}$

Los indicadores de discrimen por género que hemos señalado, colocan a la mujer como grupo todavía marginal y excluido, sufriendo las consecuencias de una sociedad patriarcal conservadora, con una socialización central sexista que la hace víctima y sobreviviente de esta situación.

En la familia, experimenta una seria situación de discrimen por maltrato conyugal, violencia familiar de todo tipo en: degradación física, emocional, sexual y hasta de seguridad escasa con riesgo de muerte (Valle, 1997). Posee escaso poder real de negociación en el matrimonio. En muchas ocasiones el cerco de la violencia familiar que la agobia la revierte contra sus hijos(as) en la crianza. En otras, experimentan toda la violencia de tipo institucional, doméstica y el maltrato a sus hijos(as) (Cabrera, 1999; Vidal, 2001; Silva, 1992). La rígida socialización de valores sexuales la mantiene responsable del "buen 
funcionamiento familiar" como valor, y de facto en múltiples responsabilidades que se le adjudican. Esto la sobrecoge física y emocionalmente, crea malestar e inseguridad en la pareja y en muchos casos es óbice de causales reales de divorcios, sobre todo en los más elevados (Muñoz y Fernández, 1988). Se ha comprobado que la violencia familiar, una vez comienza, la experimenta la mujer y los hijos(as) en un ciclo agobiante, cada vez más peligroso para ella y sus hijos. Las manifestaciones de poder y control son amplias y en diversas áreas siendo el agresor, socializado para hacer expresión de la violencia en todos sus ámbitos y la mujer socializada a aguantar la violencia, muchas veces en contra de su propia seguridad. Las manifestaciones de poder y control del agresor incluye abusos económicos, castigo mediante la privación de custodia de los hijos(as), amenazas, intimidación, «el privilegio de ser hombre», aislamiento de familiares y prohibiciones de amistades, uso de vestimentas y celos (Walter, 1996).

En la tipología de maltrato por agresión sexual conyugal una de las modalidades que tipifica la ley 54 (Violencia Doméstica) en Puerto Rico, desarrollamos una investigación cualitativa con once historias de mujeres de varias clases sociales, profesiones y ocupaciones en el año 2002 (Vidal, 2002; 1991). Encontramos como hallazgos relevantes que las mujeres entrevistadas experimentan no sólo agresión sexual de sus compañeros o esposos, sino todo tipo de agresión física, emocional y aislamiento. Sus historias de vida en la expresión de configuración de agresión sexual en su vida marital manifestaban diversión cruel, deformante y violación a la integridad y dignidad de vida humana, lo cual está prohibido en la Constitución de Puerto Rico. Algunas relataron haberlo experimentado desde meses hasta once años. Situaciones de poder y control marital gravitaban en el análisis de su configuración matrimonial y sistémica, lo cual refleja un grave problema de discrimen por género. La invisibilización de este problema de desviación es grave en Puerto Rico. Representa la modalidad menos en estadísticas de la Policía, pero no menos experimentada. Los fuertes tabúes sexuales, la indignación y humillación previenen a miles de mujeres de denunciar esta situación, en negación a su intimidad. Es necesaria más orientación, educación sobre los derechos civiles y exposición sobre la sexualidad. 
La mujer soltera jefa de familia experimenta muchas dificultades para sobrevivir, económica, social y humanamente (Vidal, 2000). Parte de sus dificultades radica en la sociedad que la discrimina y ha sido insensible a su dura tarea por desarrollarse y criar sus hijos sola cuando prevalece el status ideal de mujer casada. Más de una tercera parte de las jefaturas, son ocupadas por mujeres por razón de soltería, divorcio o viudez (Bureau of the Census, 2001). La mujer desciende económicamente con la jefatura, no empece la clase social pertenezca; lo que la puede colocar en una situación de feminización de la pobreza (Vidal, 2001). En Puerto Rico, un sector de ellas son las madres solteras adolescentes las cuales han aumentado en los últimos años. La complejidad de su situación es grave: muchas no finalizan sus estudios formales por criar a sus hijos/as; tienen gran dificultad ante la oferta y demanda en la economía; en contraposición a una economía insular que requiere más especialización y tecnificación en empleos y la competencia de otras mujeres más educadas. Los recortes federales en el sistema de bienestar social nuestro han dificultado las pocas ayudas económicas que muchas de ellas poseen. De acuerdo a la ley de Reconciliación de Responsabilidad Personal y Oportunidad Laboral, se requiere la autosuficiencia económica y un límite de cinco años para readiestrarse con recorte final a ellas. Las pensiones alimentarias bajo la ley 88 , Alimentos a Menores en Puerto Rico y reciente programa ASUME (Administración Sustituto Menores en Puerto Rico) aunque ha sido un adelanto, no llega a muchas de ellas por el alto índice de irresponsabilidad paternal que existe.

La situación de la mujer trabajadora asalariada es también delicada. Aunque se han aprobado algunas políticas públicas en legislación protectora del trabajo de la mujer, abunda el problema del discrimen por género y el hostigamiento sexual en centro de empleos. Es difícil hacer valer los derechos de las madres obreras, ascender y conseguir salarios equiparables al hombre y cumplir con la doble jornada de trabajo (Martínez, 1988; Vidal, 2001:115). La mujer retiene la responsabilidad del hogar, unidas a la laboral, lo que representa una doble jornada de trabajo, uno remunerado y otro no remunerado.

Algunas investigaciones sociales relevantes se han realizado en el tema poco abordado científicamente de las trabajadoras del sexo. Guilbe, 
(2000), realizó un estudio sobre la distribución geográfica y factores asociados a las prácticas sexuales en el área metropolitana de la capital de San Juan, Puerto Rico. Sus hallazgos corroboran nuestros supuestos sobre escasez de poder institucional y pobreza, así como factores educativas en este sector tan marginado por la sociedad.

Otra área poco estudiada es la de salud física y mental. En el Recinto de Ciencias Médicas de la Universidad de Puerto Rico se ha investigado sobre la violencia institucional y abandono de las agencias sociales de mujeres con Síndrome de Inmuno Deficiencia Asociado (SIDA) (Irizarry y Alegría, 1997). Existe otro estudio de latinas en Estados Unidos y a nivel preventivo su condición a riesgo a VIH/SIDA (Ortiz, 1995).

\section{Retos en la reformulación de política pública feminista}

Aunque hemos aprobado importante y vigorosa legislación en algunas áreas como trabajo remunerado en la mujer, la política pública a este grupo excluido adolece de:

- Una visión integral de las necesidades de la mujer en diferentes sub-áreas. Aspectos recortados en una u otra área legislativa, pero no comprehensiva. Algunas ideas desarticuladas que no recogen una visión moderna de necesidades, en la mujer, en los diferentes contextos de su vida.

- Falta de visión amplia hacia el desarrollo de una política pública que propenda al mejoramiento de las condiciones materiales, fácticas y socioeconómicas en la calidad de vida de la mujer.

La autora ha presentado un modelo de análisis de políticas públicas para la mujer. El mismo se divide en cinco grandes áreas para evaluarse:

$>$ Necesidades y problemas a atenderse en el sector femenino

$>$ Áreas a que corresponde

$>$ Evaluación de política pública 
> Legislación existente y prospectivas a nueva legislación o enmiendas adicciones a la política social

$>$ La política social, su naturaleza y relación con el trabajo social

De acuerdo a Ruiz (1997), la política social se refiere a una actividad que trasciende la práctica de la profesión, ya que la formulación de política social está por lo general en manos de otras personas que no son necesariamente trabajadores sociales y que representan las estructuras de poder.

Las políticas públicas en el área social corresponden por mandato constitucional al proceso público legislativo en su fase de formulación final y aprobación con fuerza de ley; y al ejecutivo en su aval. No obstante el proceso preparatorio de asesoría, revisión, opiniones informadas y presión corresponden a diversos sectores de la comunidad. Por ejemplo, profesionales diversos entre los que tiene una función vital el(la) trabajador(a) social por su preparación y adhesión a valores de justicia social y estrategias hacía la planificación.

Por política social denominamos planes gubernamentales y privados que de una u otra forma regulan las relaciones entre individuos, grupos, comunidades e instituciones sociales hacía finalidades de satisfacer necesidades humanas (Ruiz, 147:5). El profesional de trabajo social se visualiza desempeñándose en diversos roles en la gestión de política social, que fluctúan desde analista experto de políticas existentes, asistente de legisladores, agente de cambio en su escenario laboral y organizacional creando impacto en las políticas sociales, tanto como en los ambientes externos a su trabajo como el modelo de ideologías de política afines con la justicia social (Wyer (1991).

En la organización comunitaria, profesionales de trabajo social desempeñan un rol activo en el modelo de planificación y de acción social, y en el proceso de análisis crítico. Tanto se desempeña su militancia activa y defensa de derechos de la clientela en desventaja, como en la utilización de destrezas profesionales (Rothman, 1974). 
Concordamos con Ruiz (1997) en la responsabilidad social y profesional del trabajo social en el análisis crítico del macrosistema societal con su conjunto de prácticas, legislación en la política pública y deber de someter su conocimiento experto, destrezas y conocimientos e incidir asertivamente en nuevas políticas públicas a grupos marginados y excluidos. Dobelstien (1986) nos recuerda que los/as clientes usualmente han estado excluidos de participación activa del proceso de formulación de políticas por su escaso poder en las estructuras sociales y políticas. Por tanto, se hace necesarios nuevos enfoques de legislación que le afecten. Además de la asesoría del(la) trabajador(a) social y el trabajo en equipo multidisciplinario en coalición a ellos/as para aportar entre todos/as el conocimiento especializado necesario y hacer relevante la formulación de la política pública a grupos marginados.

En la mujer como grupo excluido se hace vigente el señalamiento de Macht y Quam (1986) para el análisis de las políticas sociales existentes, que incluye: análisis del problema ó necesidad, severidad, incidencia del grupo poblacional femenino, identificación de población atendida. Hemos integrado algunas preguntas básicas para el análisis de la política pública de Prigmore y Atherton (1986) como el nivel de satisfacción al grupo poblacional y los valores a que responde de justicia, equidad.

En el modelo que proponemos hemos incluido una evaluación interna de contenido en cada área de piezas legislativas aprobadas y vigentes y unas prospectivas para validarlas en áreas no incluidas todavía (inexistentes ó necesarias de enmendarse). Hemos partido de la premisa de que la mujer necesita una política vigorosa, integral, comprehensiva y que no sea contradictoria, que no la victimase ni propenda a su libertad individual y como grupo. No es suficiente medidas aisladas y que atienden parcialmente o insuficientemente sus necesidades.

Estas necesidades /problemas del modelo propuesto son:

1. Relación de pareja, relaciones con hijos/as, en sub-área de familia. 
2. Las dos piezas legislativas existentes Ley 54 (1989) Violencia doméstica y Ley 342 Maltrato menores (2000) son contradictorias entre si y deben armonizarse y proveer la última un marco filosófico y de servicios a (342) padres, hijos por enfoque de género. La actual estimula la parte penal, la culpabilización por maltrato y no provee vías realistas de rehabilitación y atenta contra los derechos de los padres en patria potestad. El resultado es sobrecarga de casos al Departamento de la Familia en fase la asistencial y primitiva de suspensión de custodias a menores y el Estado recargarse en costos operacionales.

3. Educación sexual, sexualidad y derechos reproductivos, entre éste el derecho al aborto.

4. Ante el incremento notable en embarazos no deseados especialmente en madres solteras y adolescentes, urge una política pública educativa y liberada en derechos reproductivos; incluyendo servicios hacía el conocimiento informado para tomar decisiones racionales en la sexualidad, alternativas ante derechos de intimidad y aborto. Hay ausencia total de pronunciamientos en política pública o planes para promulgarla. La comunidad, grupos feministas y la profesión de trabajo social deben unirse en coaliciones para presionar a legislación pertinente en esta área. Los valores religiosos, el conservadurismo y la hipocresía existente en algunos sectores estatales detienen esta legislación, aún cuando se ha evidenciado su necesidad. Existen actitudes prohibicionistas de ciertos grupos sobre el aborto que producen un impacto negativo en los derechos de muchas mujeres.

\section{$\underline{\text { Amas de casa }}$}

Este grupo ha sido invisibilizado y excluido totalmente por ausencia de política pública en legislación protectora a pesar de constituir el grupo mayoritario de mujeres. Se propone un sistema de seguridad social de retiro en determinada edad, pues es una mujer trabajadora pero sin remuneración alguna. Al contratarse los servicios por separado son costosos y especializados en nuestra sociedad, a saber: comidas en restaurantes, lavado, planchado de ropa por toda la familia, limpieza del hogar, decoración del hogar, transporte de niños/as, tutorías a niños/ as en la escuela, entre otros. 
Algunas jurisdicciones europeas ya han provisto seguros contra accidentes del hogar y otros beneficios para la ama de casa. Urge concienciar sobre el problema pues existe ignorancia en la ciudadanía sobre los problemas inherentes a esta situación de esclavitud. El sistema capitalista solo privilegia el trabajo en centros laborales y las amas de casa adolecen de reconocimiento social por su labor dedicada y agobiante.

\section{Otras áreas en la política social}

1. Trabajo renumerado, sub-área: seguridad social y legislación protectora.

Es el área en donde más legislación existe, y es vigorosa. No obstante los problemas de la mujer trabajadora fuera del hogar son muchos. Falta legislar en medidas de servicios de apoyo como centros de cuidado diurno cerca de unidades laborales como beneficios marginales, pues constituye un grave problema la protección de menores en horario donde la mujer trabaja fuera del hogar. También se necesita la provisión de recursos de orientación sobre derechos civiles para toda la población femenina, de manera que se hagan valer.

2. Educación en el género, sub-área: socialización no sexista

Es preciso legislación para hacer obligatorio en currículos escolares y universitarios la inclusión de estudios del género, al igual que legislación que controle el sexismo en medios de comunicación, lo cual es un grave problema.

3. Servicios de apoyo mujer jefa de familia; sub-área: familia, vivienda

Este sector está desprotegido por servicios y ausencia de política pública sensible que atienda necesidades reales de apoyo en: adquisición de vivienda, acceso a servicios para hijos/as gestiones en tribunales, atención de familiares enfermos, cuido de niños, recreación de la mujer.

4. Salud: sub-área tratamiento salud física y mental, servicios para adicción a drogas, condiciones como SIDA, cáncer y otras. 
Los servicios existentes bajo la reforma de salud son insuficientes, segmentados y no adaptados a necesidades especiales de la mujer. Urge una política pública integral y provisión de servicios de salud; ante aumento en condiciones emocionales, SIDA y cáncer en Puerto Rico.

a. trabajadoras del sexo: sub-áreas sexualidad, seguridad social

La política pública existente es prohibicionista y de culpabilización para toda actividad de prostitución. Urge un cambio filosófico y substancial en legalización y/o regulación de las trabajadoras del sexo en áreas de servicios de salud para prevenir el Síndrome de Inmuno Deficiencia Adquirida, y otras condiciones de salud. Además, son necesarios otros servicios de índole social en el área de la salud.

\section{Conclusiones}

La política pública para el grupo poblacional de mujeres en Puerto Rico no ha sido integral ni comprehensiva de la mayoría de sus necesidades actuales y problemas sociales asociados. La ausencia de poder real como grupo excluido la ha caracterizado en las instituciones básicas de la sociedad. Según Focault (1987) el asunto de poder es central a todas las relaciones interpersonales y sistemas sociales. Esto es particularmente visible y relevante para la mujer.

Algunas de las políticas públicas y piezas legislativas han sido, de avanzada como la legislación sobre violencia doméstica en la relación de pareja y la laboral en prohibir el hostigamiento por género, así como algunas medidas de seguridad social como ley de embrazo a (madres solteras) y en lactancia a madres obreras. Sin embargo, algunas de estas legislaciones terminan siendo políticas públicas contradictorias y plagadas de estereotipos sexistas que no resuelvan sino que agravan el problema de la mujer y la familia, como por ejemplo la Ley Maltrato Menores.

Con excepción de la ley de violencia doméstica vigente y de hostigamiento sexual en centros de empleo, que se conocen y se utilizan para un sector de la mujer, la demás legislación existente se conoce 
poco, por lo tanto no se reivindican los derechos de la mujer en la mejor parte.

La organización comunitaria hacía el apoderamiento de la mujer y la acción social en propuestas feministas representadas por la Procuraduría de la Mujer, deben agilizarse en trabajo concertados en nueva legislación coherente que realmente garanticen los derechos de la mujer.

\section{REFERENCIAS:}

Administración de Tribunales de Puerto Rico. (2001). Estadísticas. Puerto Rico: Autor.

Burgos, Nilsa y Colberg, Hielen. (1992). Madres con jefatura de familia en Puerto Rico. Río Piedras, Centro de Investigaciones Sociales, Universidad de Puerto Rico.

Cámara Representantes de Puerto Rico. (2003). Proyecto de ley para el subsidio a compra viviendas a mujeres jefes de familia.

Carpio, Jorge y Novacousky, Irene. (1999). De igual a igual: El desafio del Estado ante los nuevos problemas sociales. México: Fondo de Cultura Económica.

Carbrera, Sulemi. (1999). El impacto de la violencia en niños/as y adolescentes. Río Piedras, Puerto Rico. Instituto del Hogar Celia y Harris Bunker.

Código Penal de Puerto Rico, Art. 88.

Constitución del Estado Libre Asociado de Puerto Rico 25 de julio de 1952. Art. II secc. 1.

Departamento Familia de Puerto Rico (2002). Orden Administrativa. Puerto Rico: Estado Libre Asociado.

Dobelstein, Andrew W. (1986) Politics, economics and public welfare. Engle wood Ciffs, New Jersey Prentice Hall Inc. 
Focult, M. (1987). Power knowledge: Selected interviews. London: Harvester Wheast Shif Care.

Guilbe, Carlos. (2002). La geografía de los «amores de emergencia». Revista de Ciencias Sociales, 11, 1-28.

Irizarry, Alma y Margarita Alegría. (1997). Factores de riesgo en mujeres VIH/SIDA y agencias sociales en Puerto Rico. Río Piedras. Universidad de Puerto Rico. Recinto de Ciencias Médicas.

Ley Núm. 342. Ley Amparo de Menores en Siglo XXI, 1999, L.P.R.A.

Ley Núm. 427. Lactancia en centro de empleo, 2000, L.P.R.A.

Ley 3, Madres obreras de Puerto Rico, 1994, L.P.R.A.

Ley 88, Alimentos a Menores en Puerto Rico, 1986, L.P.R.A.

Ley 165, Enmienda a Ley Personal en el Servicio Público de Puerto Rico, 2002, L.P.R.A.

Ley 17, Prohibición hostigamiento sexual en centro de empleo, 1988, L.P.R.A.

Ley 69, Prohíbe discrimen por género en centros de empleo de Puerto Rico, 1985, L.P.R.A.

Ley 100, Prohibición de discrimen en centros de empleo, enmendada en 1972, L.P.R.A.

Ley 54, Prevención e intervención con la violencia doméstica en Puerto Rico, 1989, L.P.R.A.

Martínez, Lourdes. (1999). El hostigamiento sexual en los centros de empleo. Río Piedras. Universidad de Puerto Rico. Centro de Investigaciones Sociales.

Match, Mary y Jean Guan. (1986). Social Work: An introduction. Columbus, Ohio: Charles Merriel Publishing Co. 
Muñoz, Marya y Edwin Fernández. (1988). Divorcio, persona y sociedad. Río Piedras: Editorial Huracán.

Ortiz, Blanca. (1999). Estrategias de prevención de VIH/SIDA entre mujeres latinas. New York: New York State Psychiatrics Institute, Columbus University.

Pascall, G. (1986). Social policy: a feminist analysis. London: Tavistock Publication.

Policía de Puerto Rico (2001). Informe estadístico. San Juan, Puerto Rico: Autor.

Prigmore, Charles S. y Charles Altherton. (1986). Social welfare policy. Lexington. Mass: D.C. Health and Company.

Rotman, Jack. (1974). Three models of community organization practice en Cix, F. et all. Eds. Stategies of community organization Peacock, Ill: 26 - 27.

Ruiz, Magali. (1997). Organización de la comunidady política social. Un compendio. Río Piedras: Editoral Edil.

Russell, M. (1984). In counseling women: The feminist approach. Springfield: Charles Thomas Publis.

Segarra, Hielen. (2000). La reforma del bienestar social y el mercado federal en Puerto Rico. Diálogo. Río Piedras, Universidad de Puerto Rico.

Silva, Ruth. (1982). Amas de casa en la fuerza laboral: el lenguaje como mediación ideológica en la conciencia. Río Piedras, Puerto Rico: Centro de Investigaciones Sociales, Universidad de Puerto Rico.

Silva, Ruth y Lourdes Martínez, et al. (1990). Hay amores que matan: La violencia contra las mujeres en la vida conyugal. Río Piedras: Editorial Huracán.

Título VII Ley Federal Derechos Civiles. (1964). Estados Unidos de Norteamérica 42 U.S.C. secc. 2000. 
Tribunal Superior de Estados Unidos de América. (1973). Row vs. Wae.

Valle, Diana y Albite, William. (1998). Violencia en la familia: Una perspectiva crítica. San Juan. Ediciones Familia y Comunidad.

US Bureau of the Census. (2001). Census of the population of Puerto Rico. Washington: Autor.

Vidal, Zuleika. (2001). La mujer en Puerto Rico: Retos, demandas y perspectivas ante un nuevo milenio. Río Piedras, Puerto Rico: Editorial Edil.

Walker, Leonore. (1996). Issues and dilemmas in domestic violence. Washington, D.C.: American Psyshologic Association.

Wyers, Norman L. (1991). Policy practice in social work. Models and issues. Journal of Social Work Education, 27. (Otoño), 243-46. 
